\title{
EFEITO DO COMPRIMENTO DO CORPO-DE-PROVA NA VELOCIDADE ULTRA-SÔNICA EM MADEIRAS ${ }^{1}$
}

\author{
Fabiana Goia Rosa de Oliveira², Karla Peitl Miller ${ }^{3}$, Marcela Candian³ e Almir Sales ${ }^{3}$
}

\begin{abstract}
RESUMO - O presente artigo apresenta um estudo sobre a variação da velocidade ultra-sônica longitudinal em função do comprimento da peça de madeira. As espécies utilizadas foram: pinus caribaea (Pinus caribaea var. caribaea) eucalipto citriodora (Eucalyptus citriodora), eucalipto grandis (Eucalyptus grandis) e jatobá (Hymenaea sp). Utilizou-se equipamento de ultra-som com transdutores exponenciais de $22 \mathrm{kHz}$. Foram feitas medições do tempo de propagação das ondas ultra-sônicas, com o comprimento variando de $300 \mathrm{~cm}$ a 10 cm e mantendo-se a seção transversal constante de $12 \mathrm{~cm}$ x $5 \mathrm{~cm}$. Os resultados apontaram que ocorre variação da velocidade em função da distância percorrida e do comprimento de onda $(\lambda)$ utilizado. Conclui-se que adequar a freqüência do transdutor com o comprimento da peça é essencial para a determinação correta da velocidade ultra-sônica em madeiras.
\end{abstract}

Palavras-chave: Madeira, ultra-som e comprimento de onda.

\section{EFFECT OF THE SIZE OF THE SPECIMEN ON ULTRASONIC VELOCITY}

\begin{abstract}
This paper reports on a study of the variation of ultrasonic velocity with the length of the specimen of pinus caribaea (Pinus caribaea var. caribaea) eucalipto citriodora (Eucalyptus citriodora), eucalipto grandis (Eucalyptus grandis) and jatobá (Hymenaea sp). Ultrasonic measurements were taken with an experimental apparatus having $22 \mathrm{kHz}$ transducers. Tests were carried out with length from $300 \mathrm{~cm}$ to $10 \mathrm{~cm}$ and constant cross-section $(12 \mathrm{~cm} \times 5 \mathrm{~cm})$. The results showed that velocity of ultrasonic waves is sensitive to changes in the distance between transducers and wavelength $(\lambda)$. The adjustment between the frequency of transducer and the length of the specimen is essential in order to obtain correct measurements of ultrasonic velocity in wood.
\end{abstract}

Keywords: Wood, ultrasound and wavelength.

\section{INTRODUÇÃO}

Os avanços tecnológicos têm permitido melhorar o aproveitamento das propriedades da madeira e de seus derivados, criando novos produtos e ampliando sua aplicação, o que possibilitará que a madeira seja um material ainda mais versátil e empregado, já que seu aproveitamento não danifica o meio ambiente.

Dentre esses avanços, pode-se destacar a técnica de ultra-som, importante ferramenta com potencial para melhorar a qualidade e a competitividade da madeira. Mas, para isso, faz-se necessária a compreensão do fenômeno de propagação da onda ultra-sônica no material, através do estabelecimento de relações entre as diversas variáveis envolvidas (OLIVEIRA, 2001).

Segundo Bucur e Böhnke (1994), os fatores que influenciam a propagação de ondas ultra-sônicas na madeira são: propriedades físicas do substrato, características geométricas da espécie, condições do

\footnotetext{
${ }^{1}$ Recebido em 10.05.2004 e aceito para publicação em 10.11.2005.

${ }^{2}$ LaMEM, Escola de Engenharia de São Carlos, USP, Av.Trabalhador Sãocarlense, 400, 13566-590São Carlos-SP. E-mail: <fabiana@sc.usp.br).

${ }^{3}$ DeCiv, Universidade Federal de São Carlos - Rodovia Washington Luis, km 235, 13565-905 São Carlos-SP. E-mail: <almir@power.ufscar.br>.
} 
meio (temperatura e umidade) e procedimento utilizado para a tomada das medidas.

A geometria do corpo-de-prova pode ter efeito decisivo na determinação da velocidade ultra-sônica e também no modo de propagação da onda sonora. Conforme a onda sonora interage com os contornos do corpo-de-prova, forças de tração, compressão ou cisalhamento distorcem e interferem na onda, dificultando a precisão das medições do tempo de propagação (BROWN, 1997).

As condições para a preparação satisfatória das amostras dependem da magnitude da atenuação da onda ultra-sônica na madeira. Um limite mínimo para o tamanho do corpo-de-prova é necessário, pois a onda ultra-sônica deve possuir a característica de onda plana em um meio infinito. O tamanho mínimo deve ser estabelecido experimentalmente, porque não há um bom critério teórico para isso (BUCUR, 1995). Amostras maiores apresentam maior exatidão nos resultados devido ao maior tempo de propagação do sinal ultra-sônico na madeira, permitindo maior interação da onda com os constituintes da madeira.

Em razão da relação entre as dimensões do corpode-prova e o comprimento de onda, podem-se ter diferentes tipos de propagação de ondas ultra-sônicas. As ondas de volume ocorrem quando as dimensões do corpo-de-prova são maiores se comparadas com o comprimento de onda e podem ser longitudinais ou transversais, dependendo do tipo de transdutor utilizado.

Segundo Bucur (2003), quando uma das dimensões do corpo-de-prova se aproxima do comprimento de onda, a propagação ocorre por meio de ondas de superfície, as quais são mais complexas e com maior atenuação. As ondas de superfície podem ser divididas em ondas de placa, caracterizadas pelo efeito fronteira, com maior amortecimento devido à mudança da impedância acústica e ondas Rayleigh, nas quais a trajetória da onda é elíptica e paralela à direção de propagação.

A preparação adequada da amostra é essencial para a determinação de velocidades ultra-sônicas. Das três direções principais de propagação, podese afirmar que a velocidade na direção longitudinal é a mais afetada pela variação da geometria do corpode-prova (BUCUR, 1995).
A falta de homogeneidade da madeira limita a exatidão das medidas de velocidade de propagação. Se uma amostra apresentar fissuras internas, o pulso ultra-sônico será atenuado por dispersão nas interfaces, mas se a dimensão da descontinuidade for muito menor que o comprimento de onda, a alteração do pulso será pequena, permitindo bons resultados (BEALL, 2002).

De acordo com Berndt et al. (2000), a influência da variabilidade natural das espécies na velocidade e atenuação pode ser analisada pela escolha de uma freqüência da fonte na qual o comprimento de onda no material fique aproximadamente entre a dimensão máxima dos elementos anatômicos e a dimensão mínima da amostra.

Puccini et al. (2001) realizaram ensaios variando o comprimento de tábuas de Pinho do Paraná com propagação de ondas de superfície e de compressão. Os resultados indicaram que a velocidade se torna constante quando o comprimento da tábua é maior que $7 \lambda$ e $2,5 \lambda$, respectivamente, para as ondas de superfície e de compressão.

É nesse contexto que, compreendendo-se as relações entre os fatores que afetam as condições e os parâmetros físicos da técnica de ultra-som, pode-se melhorar seu potencial de aplicação.

O objetivo do presente trabalho foi avaliar a influência da relação entre o comprimento do corpo-de-prova e o comprimento de onda nas espécies de pinus caribaea, eucalipto grandis, eucalipto citriodora e jatobá na velocidade ultra-sônica longitudinal, por meio da variação do comprimento das peças.

\section{MATERIAL E MÉTODOS}

As espécies utilizadas foram: pinus caribaea (Pinus caribaea var. caribaea), eucalipto citriodora (Eucalyptus citriodora), eucalipto grandis (Eucalyptus grandis) e jatobá (Hymenaea sp). As peças apresentavam teor de umidade em torno de $12 \%$.

Foram utilizadas 12 vigas para cada espécie, com comprimento inicial de $300 \mathrm{~cm}$ e seção transversal de $12 \mathrm{~cm}$ x $5 \mathrm{~cm}$. O comprimento das vigas foi reduzido continuamente até $10 \mathrm{~cm}$.

Para cada variação do comprimento das vigas, foram feitas medições do tempo de propagação da onda ultra-sônica longitudinal. O equipamento de ultra-som foi utilizado com transdutores exponenciais de $22 \mathrm{kHz}$. 
A relação entre a distância percorrida pela onda ultra-sônica (L) e o comprimento de onda $(\lambda)$ variou aproximadamente entre 15 e 0,5 . O comprimento de onda foi calculado com a seguinte equação:

$$
\lambda=\mathrm{V} / \mathrm{f}
$$

em que:

$\mathrm{V}=$ velocidade longitudinal para o comprimento inicial $(\mathrm{m} / \mathrm{s})$; e

$$
\mathrm{f}=\text { freqüência do transdutor }(\mathrm{kHz}) \text {. }
$$

Com os valores dos tempos de propagação e tendo a distância percorrida em cada intervalo, calcularamse as velocidades de propagação de cada espécie.

\section{RESULTADOS E DISCUSSÕES}

Na Tabela 1 estão apresentados os valores das velocidades médias, máximas e mínimas e os coeficientes de variação das espécies estudadas.

Com base nos resultados apresentados, verificouse que nas espécies estudadas a velocidade ultra-sônica longitudinal mostrou-se sensível à variação da relação entre o comprimento da peça e o comprimento de onda utilizado.

Os resultados apontaram que existe um ponto crítico na relação entre comprimento da peça e comprimento de onda $(L / \lambda)$, a partir do qual a velocidade não é mais afetada, ou seja, para a freqüência utilizada existe um comprimento mínimo da peça, equivalente a alguns comprimentos de onda, para que a velocidade obtida não sofra interferências significativas.

A determinação do ponto crítico foi feita em cada espécie, através de regressões lineares em ambos os trechos (acima e abaixo do ponto crítico), a partir do gráfico das velocidades médias em cada conjunto de 12 vigas.

Tabela 1 - Velocidades médias, máximas e mínimas e coeficientes de variação.

Table 1 - Average, maximum and minimum velocities and coefficient of variation

\begin{tabular}{lcccc}
\hline & $\begin{array}{c}\mathrm{V}_{\text {média }} \\
(\mathrm{m} / \mathrm{s})\end{array}$ & $\begin{array}{c}\mathrm{V}_{\text {mín }} \\
(\mathrm{m} / \mathrm{s})\end{array}$ & $\begin{array}{c}\mathrm{V}_{\text {máx }} \\
(\mathrm{m} / \mathrm{s})\end{array}$ & $\begin{array}{c}\mathrm{CV} \\
(\%)\end{array}$ \\
\hline Pinus caribaea & 3873 & 2745 & 4706 & 12,5 \\
Euc. grandis & 4724 & 3333 & 5405 & 9,3 \\
Euc. citriodora & 4660 & 3544 & 5195 & 8,3 \\
Jatobá & 4283 & 2857 & 5415 & 13,4 \\
\hline
\end{tabular}

A seguir são apresentados os gráficos da velocidade média longitudinal em função da variação do comprimento das peças de madeira, em que L é o comprimento da peça e $\lambda$, o comprimento de onda utilizado para a freqüência de $22 \mathrm{kHz}$. O comprimento de onda, calculado a partir da média da velocidade inicial nas espécies em estudo, de acordo com a equação (1), foi em torno de $20 \mathrm{~cm}$.

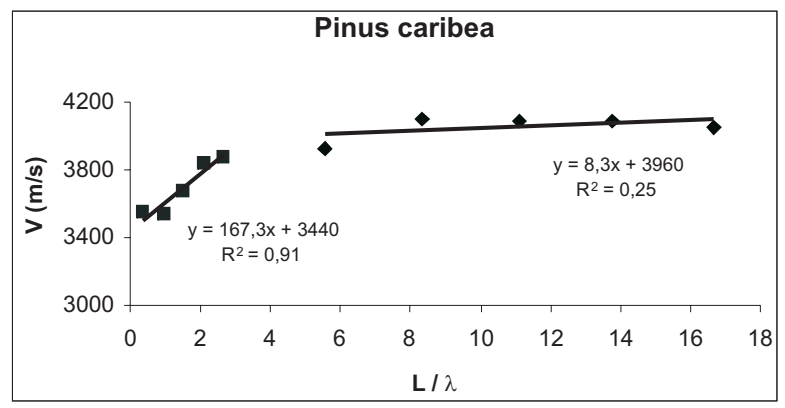

Figura 1 - Velocidade média em função da relação $L / \lambda$ pinus caribaea.

Figure 1 - Mean velocity as a function of $L / \lambda$ - pinus caribaea.

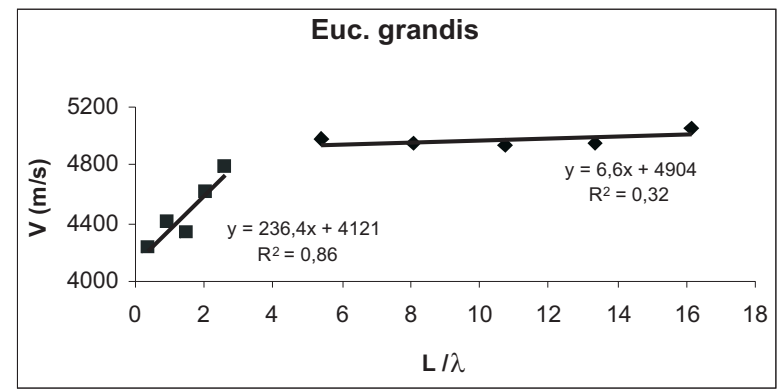

Figura 2 - Velocidade média em função da relação L/ $\lambda$ eucalipto grandis.

Figure 2 - Mean velocity as a function of $L / \lambda$ - eucalipto grandis.

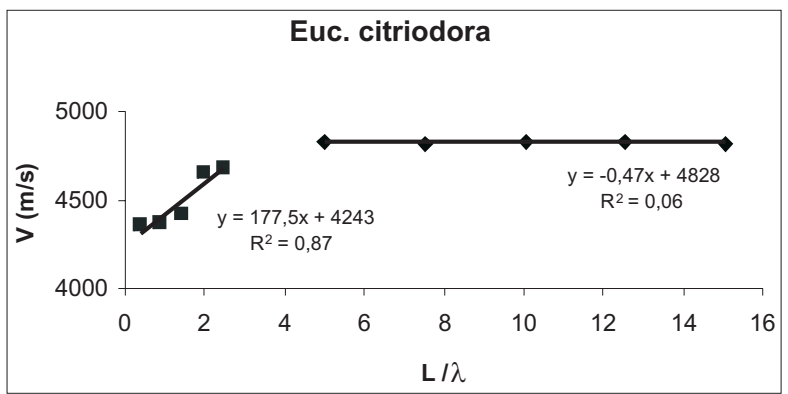

Figura 3 - Velocidade média em função da relação L/ $\lambda$ eucalipto citriodora.

Figure 3 - Mean velocity as a function of $L / \lambda$ - eucalipto citriodora.

R. Árvore, Viçosa-MG, v.30, n.1, p.141-145, 2006 
As equações obtidas e os coeficientes de determinação estão apresentados na Tabela 2, assim como o ponto crítico $\mathrm{L} / \lambda$ em cada espécie estudada.

Os coeficientes de determinação $\left(\mathrm{R}^{2}\right)$ obtidos no trecho acima do ponto crítico em cada espécie estudada foram baixos, podendo-se afirmar, assim, que a velocidade não é mais afetada pela razão $L / \lambda$. Nas espécies avaliadas nesta experimentação, o valor crítico ocorreu quando $\mathrm{L} / \lambda$ foi igual a 3 .

Os resultados estão de acordo com a literatura pesquisada (BUCUR, 1995; PUCCINI et al., 2001;

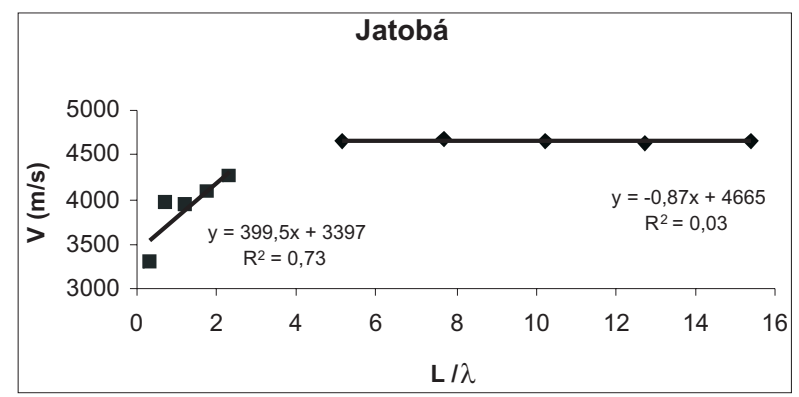

Figura 4 - Velocidade média em função da relação $L / \lambda$ jatobá.

Figure 4 - Mean velocity as a function of $L / \lambda$-jatobá.
BARTHOLOMEU et al., 2003) e demonstraram que existe um ponto crítico na relação entre comprimento da peça e comprimento de onda $(\mathrm{L} / \lambda)$, a partir do qual a velocidade não é mais afetada, ou seja, para a freqüência utilizada, existe um comprimento mínimo da peça, equivalente a alguns comprimentos de onda, para que a velocidade obtida não tenha interferências.

\section{CONCLUSÕES}

Com base nos resultados apresentados, pode-se concluir que nas espécies estudadas, pinus caribaea, eucalipto grandis, eucalipto citriodora e jatobá, a velocidade ultra-sônica longitudinal mostrou-se sensível à variação da relação entre o comprimento da peça e o comprimento de onda utilizado.

Os resultados indicaram que existe um ponto crítico na relação L/l, a partir do qual não ocorre mais interferência na velocidade obtida. Neste estudo, observou-se que é necessário o corpo-de-prova ser três vezes maior que o comprimento de onda.

Conclui-se que é necessário adequar a freqüência do equipamento com o tamanho do corpo-de-prova, para que se obtenha a correta velocidade ultra-sônica em madeiras.

Tabela 2 - Equações das regressões lineares (determinação do ponto crítico $L / \lambda$ )

Table 2 - Linear regressions (determination of critical point $L / \lambda$ )

\begin{tabular}{lcccc}
\hline & \multicolumn{2}{c}{ Abaixo Ponto Crítico } & \multicolumn{2}{c}{ Acima Ponto Crítico } \\
\cline { 2 - 4 } & \multicolumn{2}{c}{ Equação } & $\mathrm{R}^{2}$ & \multicolumn{2}{c}{ Equação } & $\mathrm{R}^{2}$ \\
\hline Pinus caribaea & $\mathrm{y}=167,3 \mathrm{x}+3440$ & 0,91 & $\mathrm{y}=8,3 \mathrm{x}+3960$ & 3 \\
Euc. grandis & $\mathrm{y}=236,4 \mathrm{x}+4121$ & 0,86 & $\mathrm{y}=6,6 \mathrm{x}+4904$ & 0,32 \\
Euc. citriodora & $\mathrm{y}=177,5 \mathrm{x}+4243$ & 0,87 & $\mathrm{y}=-0,47 \mathrm{x}+4828$ & 0,06 \\
Jatobá & $\mathrm{y}=399,5 \mathrm{x}+3397$ & 0,73 & $\mathrm{y}=-0,87 \mathrm{x}+4665$ & 0,03 \\
\hline
\end{tabular}

\section{AGRADECIMENTOS}

À Fundação de Amparo à Pesquisa do Estado de São Paulo (FAPESP) e ao Laboratório de Madeiras e Estruturas de Madeira (LaMEM), da Escola de Engenharia da Universidade de São Paulo, em São Carlos.

\section{REFERÊNCIAS BIBLIOGRÁFICAS}

BARTHOLOMEU, A.; GONÇALVES, R.; BUCUR, V. Dispersion of ultrasonic waves in eucalyptus lumber as a function of the geometry of boards. Scientia Forestalis, n. 63, p. 235-240, 2003.
BEALL, F. C. Overview of the use of ultrasonic technologies in research on wood properties. Wood Science and Technology. v. 36, p.197-212, 2002.

BERNDT, H.; SCHNIEWIND, A. P.; JOHNSON, G. C. Ultrasonic energy propagation through wood: where, when, how much. In: SYMPOSIUM NDT OF WOOD, 12., 2000, Sopron. Proceedings... Sopron: University of Western Hungary, 2000. p. 57-65. 
BROWN, A. E. Rationale and summary of methods of determining ultrasonic properties of materials at Lawrence National Laboratory. UCRL - ID - 119958. New York: CRC Press, 1997.

BUCUR, V.; BÖHNKE, I. Factors affecting ultrasonic measurements in solid wood.

Ultrasonics, v. 32, n. 5, p. 385-390, 1994.

BUCUR, V. Acoustics of wood. New York: CRC Press,1995.

BUCUR, V. Nondestructive

characterization and imaging of wood. New York: Springer-Verlag, 2003. p. 324.
OLIVEIRA, F. G. R. Estudo de propriedades mecânicas de dicotiledôneas por meio de ensaio não-destrutivo utilizando equipamento de ultra-som. 2001. 104f. Dissertação (Mestrado em Engenharia de Materiais) - Universidade de São de São Paulo, 2001.

PUCCINI, C. T.; GONÇALVES, R.; BALLARIN, A. W. Dispersão de ondas de ultra-som em peças de madeira serrada de Pinho do Paraná em função do comprimento. In: CONGRESSO NACIONAL DE ENSAIOS NÃO-DESTRUTIVOS, 20., 2001. São Paulo. Anais... São Paulo: 2001. CD-ROM. 